\title{
Surface characterization of steel/steel contact lubricated by PAO6 with novel black phosphorus nanocomposites
}

\author{
Zhiheng LUO ${ }^{1}$, Jingyuan YU ${ }^{1}$, Yufu XU ${ }^{1, *}$, He XI ${ }^{1}$, Gang CHENG ${ }^{1}$, Lulu YAO ${ }^{2}$, Ruhong SONG ${ }^{1}$, Karl D DEARN ${ }^{3}$ \\ ${ }^{1}$ Institute of Tribology, School of Mechanical Engineering, Hefei University of Technology, Hefei 230009, China \\ ${ }^{2}$ School of Chemistry and Chemical Engineering, Hefei University of Technology, Hefei 230009, China \\ ${ }^{3}$ Mason Institute of Tribology, School of Engineering, University of Birmingham, Edgbaston, Birmingham B152TT, United Kingdom \\ Received: 01 November 2019 / Revised: 02 January 2020 / Accepted: 01 February 2020 \\ (C) The author(s) 2020.
}

\begin{abstract}
In the present work, two types of novel nano additives, titanium sulfonate ligand/black phosphorus $\left(\mathrm{TiL}_{4} / \mathrm{BP}\right)$ and titanium dioxide/black phosphorus $\left(\mathrm{TiO}_{2} / \mathrm{BP}\right)$ nanocomposites, were prepared. The tribological behavior of the steel/steel friction pairs lubricated by polyalphaolefins type 6 (PAO6) containing the nanocomposites under boundary lubrication was studied. The worn surfaces were analyzed using modern surface techniques. The experimental results show that the rubbed surfaces became smooth and showed little wear with the addition of the nanocomposites. $\mathrm{TiO}_{2} / \mathrm{BP}$ nanocomposites can significantly improve the lubricity of $\mathrm{BP}$ nanosheets under high contact stress. The synergistic roles of the load-bearing abilities and rolling effect of $\mathrm{TiO}_{2}$ nanoparticles, the slip induced by the BP with its layered structure, and the establishment of a tribofilm on the sliding interface are the basis of the tribological mechanisms.
\end{abstract}

Keywords: surface characterization; black phosphorus (BP) nanosheets; nano titanium dioxide; boundary lubrication; steel/steel contact

\section{Introduction}

Steel/steel contact is one of the most common friction pairs in modern mechanical equipment. The use of lubricants can save energy and enhance the efficiency of mechanical equipment by reducing the friction and wear of the critical steel/steel friction pairs [1]. Therefore, it is vital to upgrade the lubrication performance of lubricants for friction pairs in mechanical instruments. Current research has found that many nanoparticle-based lubricant additives can signifycantly enhance the lubricities of lubricants because of the unique properties of these nanoparticles [2-4]. However, most of the current research has focused on traditional lubricants such as molybdenum disulfide [5] or newly-appeared graphene [6]. The development of new-additives and the surface characterization of steel/steel contact is urgently needed but is not well studied.

Phosphorus is an active element during sliding and has excellent lubricating properties, especially incorporated in zinc dialkyldithiophosphate (ZDDP) [7-9], commonly incorporated in lubricating oils for mechanical equipment. Phosphorus, itself, is the eleventh most abundant element in the earth's crust. Moreover, phosphorus-containing additives can decrease the wear of contacts through the formation of a tribofilm on the sliding interfaces [10], thereby improving the service life of mechanical equipment.

Recently, black phosphorus (BP) has gained significant interest owing to its unique multi-layered structures [11-14]. Wang et al. [11] investigated the tribological behavior of BP nanosheets in liquid paraffin. The research results indicate that the primary mechanism for the antifriction and lubrication role of $\mathrm{BP}$ nanosheets was the interlaminar slip between the

* Corresponding author: Yufu XU, E-mail: xuyufu@hfut.edu.cn 
nanosheets and the formation of a tribofilm over the frictional surfaces. Lv et al. [12] studied the friction and wear properties of BP mixed with PTFE-based composites. Their experimental results show that BP might be able to decrease the wear rate of the composites significantly. The underlying lubricating mechanism for BP is the formation of transfer films at the frictional interface. Nevertheless, in a recent study of BP nanosheets [15], it was found that the lubricity of BP nanosheets under high contact stress conditions is insufficient, restricting its tribological application in the most commonly used steel/steel contact applications under harsh conditions, such as heavy equipment.

Combining BP with other lubricating components is a promising way to improve the lubricity of the nanosheets under high contact stress. Titanium dioxide is a common mineral with excellent lubricating and self-healing properties under certain contact stress conditions [16-18]. In this work, experiments measured and charateristerised the tribological properties of two types of novel BP nanocomposites, titanium sulfonate ligand/black phosphorus $\left(\mathrm{TiL}_{4} / \mathrm{BP}\right)$ and titanium dioxide/black phosphorus $\left(\mathrm{TiO}_{2} / \mathrm{BP}\right)$, under steel/steel contact surfaces and lubricated with polyalphaolefins type 6 (PAO6).

\section{Experimental details}

\subsection{Materials}

The lubricant was PAO6, purchased from Shandong Yousuo Chemical Reagent Co., Ltd., China. BP nanosheets were prepared from red phosphorus using ball milling techniques based on previous work [15]. Titanium tetraisopropoxide was the product of Shanghai Maclean Biochemical Technology Co., Ltd., China. Also, all the chemicals used in the experiments were of analytical reagent grade, and in their initial states without further treatment. Table 1 defines the underlying physical parameters of the steel/steel contact.

\subsection{Synthesis of $\mathrm{TiO}_{2} / \mathrm{BP}$ and $\mathrm{TiL}_{4} / \mathrm{BP}$ nano- composites}

$\mathrm{TiO}_{2} / \mathrm{BP}$ nanocomposites were synthesized with a sol-gel method [19]. A typical synthesis is as follows: Initially, $3 \mathrm{mg}$ of BP nanosheets is dispersed in $50 \mathrm{~mL}$ of absolute ethanol. Then, $1 \mathrm{~mL}$ of acetic acid is placed into absolute ethanol, after which the mixture is evenly stirred. Next, $10.7 \mathrm{mg}$ of titanium tetraisopropoxide is added to $10 \mathrm{~mL}$ of absolute ethanol, and is then poured into the former mixed solution, combined with intensive stirring. The re-mixed solution is then sonicated for $30 \mathrm{~min}$, after which $1 \mathrm{~mL}$ of distilled water (DI) is introduced (drop by drop), followed by stirring for $1 \mathrm{~h}$. Finally, the $\mathrm{TiO}_{2} / \mathrm{BP}$ nanocomposites are separated by centrifugation and dried at $60^{\circ} \mathrm{C}$.

The preparation of $\mathrm{TiL}_{4} / \mathrm{BP}$ nanocomposites referred to the previous work [20]. In a typical synthesis, $15.2176 \mathrm{~g}$ of $\mathrm{p}$-toluenesulfonic acid is dissolved in absolute ethanol, and $5.6844 \mathrm{~g}$ of titanium tetraisopropoxide is introduced (drop-by-drop) into solution. The mixed solution is stirred at $50{ }^{\circ} \mathrm{C}$, reacting for $3 \mathrm{~h}$. Titanium benzenesulfonate is then obtained after removing the solvent by rotary evaporation. Subsequently, $3 \mathrm{mg}$ of titanium benzenesulfonate is added into a $50 \mathrm{~mL}$ BP N-methyl-2-pyrrolidinone solution and stirred for $15 \mathrm{~h}$ in the dark. Finally, solid $\mathrm{TiL}_{4} / \mathrm{BP}$ nanocomposites are obtained after centrifugation and drying.

\subsection{Tribological tests}

Before the tribological tests, the two types of as-prepared nanocomposites, $\mathrm{TiO}_{2} / \mathrm{BP}$ and $\mathrm{TiL}_{4} / \mathrm{BP}$, were dispersed in the PAO6 lubricant with mass concentrations of $0 \%, 0.005 \%, 0.01 \%, 0.05 \%$, and $0.2 \%$, respectively, with oleic acid as the dispersant. The lubricants were then treated by ultrasound for $30 \mathrm{~min}$ before the tribological tests, conducted using a CFT-1 ball-on-disk tribometer (Jinan Yihua Tribological Test Technology Co., Ltd.). The dispersions were kept stable during the entire sliding process. Figure 1 shows

Table 1 Parameters of the steel/steel contact.

\begin{tabular}{cccccc}
\hline Specimen & Material & Diameter $(\mathrm{mm})$ & Hardness $(\mathrm{HV})$ & Roughness $(\mu \mathrm{m})$ & Thickness $(\mathrm{mm})$ \\
\hline Upper ball & GCr15 & 6 & $660-880$ & 0.05 & - \\
Lower disk & GCr15 & 30 & $190-210$ & 0.20 & 3 \\
\hline
\end{tabular}




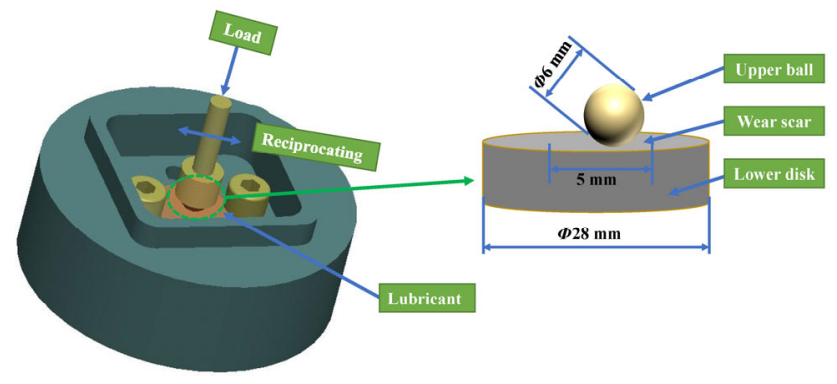

Fig. 1 Schematic of tribological tests.

the test rig schematic and the Table 2 lists the test parameters.

The film thickness ratio confirmed the lubrication regime. According to Hertz's theory, the contact stress of a ball-on-disk contact was [21]:

$$
\begin{gathered}
P=\frac{4 W}{\pi a^{2}} \\
a=2\left(\frac{2}{3} \times \frac{W R}{E^{\prime}}\right)^{\frac{1}{3}}
\end{gathered}
$$

where $P$ and $a$ represent the Hertz contact stress and contact diameter; $W$ is the normal pressure $(W=8,20$, and $30 \mathrm{~N}) ; R$, the equivalent radius of curvature $(R=$ $3 \mathrm{~mm}) ; E^{\prime}$, the finite elastic modulus $\left(E^{\prime}=233 \mathrm{GPa}\right)$. According to Eqs. (1) and (2), the contact stresses of the steel/steel contact were 1.67, 2.34, and $2.36 \mathrm{GPa}$, respectively for each pressure.

The Hamrock and Dowson formula estimates the film thickness ratio $\lambda$ using the formula of the minimum thickness of oil film [2].

$$
\begin{gathered}
h_{\min }=3.63 R \frac{G^{* 0.49} U^{* 0.68}}{W^{* 0.073}}\left(1-\mathrm{e}^{-0.068 k}\right) \\
\lambda=\frac{h_{\min }}{\left(R \mathrm{a}_{1}^{2}+R \mathrm{a}_{2}^{2}\right)^{\frac{1}{2}}}
\end{gathered}
$$

where $G^{*}=\alpha E^{\prime} ; U^{*}=\eta_{0} U / E^{\prime} R ; W^{*}=W / E^{\prime} R^{2} ; U$ represents

Table 2 Tribological testing conditions.

\begin{tabular}{ccc}
\hline Testing condition & Unit & Value \\
\hline Sliding speed & $\mathrm{m} / \mathrm{s}$ & 0.083 \\
Stroke length & $\mathrm{mm}$ & 5 \\
Loads & $\mathrm{N}$ & $8,20,30$ \\
Sliding time & $\mathrm{min}$ & 15 \\
Temperature & ${ }^{\circ} \mathrm{C}$ & 25 \\
\hline
\end{tabular}

the sliding velocity $(U=83 \mathrm{~mm} / \mathrm{s}) ; \alpha$, the pressureviscosity coefficient; $\eta_{0}$, the dynamic viscosity $\left(\eta_{0} \approx\right.$ $\left.30.5 \times 10^{-3}(\mathrm{~N} \cdot \mathrm{s}) / \mathrm{m}^{2}\right) ; k$, the elliptical parameter; $R a$, the surface roughness of the upper and lower samples $\left(R a_{1}=50 \mathrm{~nm}, R a_{2}=200 \mathrm{~nm}\right)$. According to the calculated results, the maximum lambda ratio is 0.216 for all the tests, suggesting that all the sliding tests remain within the boundary lubrication regime.

\subsection{Characterization}

Microscopic confocal laser Raman spectroscopy (HR Evolution) determined the structure of the BP powder and BP nanosheets. A JEOL EM-2100F high-resolution transmission electron microscope (TEM) and a digital camera characterized the microscopic and macromorphologies of BP nanocomposites and their dispersions, respectively. The Keyence VK-X1003 Dimension laser scanning microscopy was selected to obtain the surface profile. The SU8020 high-resolution scanning electron microscopy (SEM) was employed to observe the microscopic morphologies of the worn surfaces after sliding. The chemical components of the rubbed surfaces were detected using the ESCALAB 250Xi X-ray photoelectron spectroscopy (XPS).

\section{Results and discussion}

\subsection{Characterization of $\mathrm{TiO}_{2} / \mathrm{BP}$ and $\mathrm{TiL}_{4} / \mathrm{BP}$ nano- composites}

Figure 2 shows the Raman spectra of BP nanosheets (BP-ns) and BP nanocomposites. As shown in Fig. 2, all three characteristic peaks of BP occurred at $360 \mathrm{~cm}^{-1}$ $\left(\mathrm{A}_{\mathrm{g}}{ }^{1}\right), \sim 440 \mathrm{~cm}^{-1}\left(\mathrm{~B}_{2 \mathrm{~g}}\right)$, and $\sim 466 \mathrm{~cm}^{-1}\left(\mathrm{~A}_{\mathrm{g}}{ }^{2}\right)$, respectively. The $\mathrm{BP}$ nanosheets had higher peak intensity ratios of $\mathrm{A}_{\mathrm{g}}{ }^{2}$ to $\mathrm{B}_{2 \mathrm{~g}}$ than the bulk BP powder, indicating the successful reduction of the layers [15]. However, from Fig. 2(a) the characteristic peaks of $\mathrm{TiL}_{4} / \mathrm{BP}$ had a redshift compared to those of the BP nanosheets, which may have resulted from restricted vibration of the phosphorus atoms, caused by the affiliated surface $\mathrm{TiL}_{4}$ [20]. However, the $\mathrm{TiO}_{2}$ had little influence on the reduction of the corresponding Raman scattering energy owing to its small particle size. From Fig. 2(b), the characteristic peaks of the $\mathrm{BP}$ nanosheets and $\mathrm{TiO}_{2}$ $[21,22]$ are present in the $\mathrm{TiO}_{2} / \mathrm{BP}$ nanocomposites, which agrees well with their constituent components. 

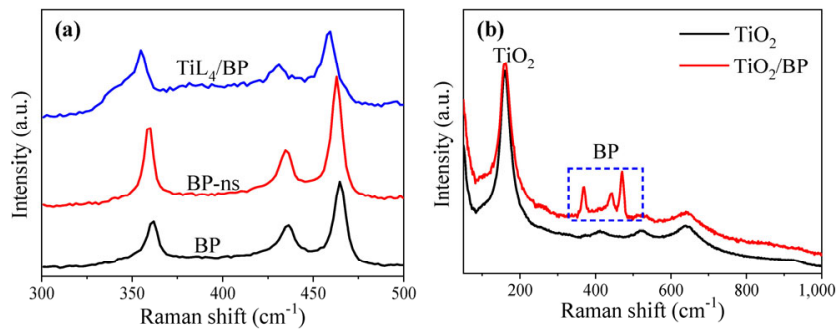

Fig. 2 Raman spectra of the nanocomposites: (a) $\mathrm{TiL}_{4} / \mathrm{BP}$ and (b) $\mathrm{TiO}_{2} / \mathrm{BP}$.

Figure 3 shows the TEM images of BP nanocomposites and the digital photos PAO6 containing different mass concentrations of the nanocomposites, after standing for $48 \mathrm{~h}$. Note the typical thin and lamellar heterostructures of the $\mathrm{TiL}_{4} / \mathrm{BP}$ nanocomposites (Fig. 3(a)), the composite sheets had a dimension of approximately $250 \mathrm{~nm} \times 300 \mathrm{~nm}$. As shown in Fig. 3(c), many $\mathrm{TiO}_{2}$ nanoballs were well dispersed over the surface of the BP nanosheets (of dimensions approximately $300 \mathrm{~nm} \times 500 \mathrm{~nm}$ ). All the nanocomposites with different concentrations, shown in Figs. 3(b) and 3(d), exhibit excellent dispersive effects after standing for $48 \mathrm{~h}$, except for $0.2 \%$ concentration $\mathrm{TiL}_{4} / \mathrm{BP}$. Figures 2 and 3 confirm successful preparation of the BP nanocomposites stable dispersions.

\subsection{Tribological properties}

Figure 4 shows the average coefficients of friction (COFs) of steel/steel contact lubricated by PAO6

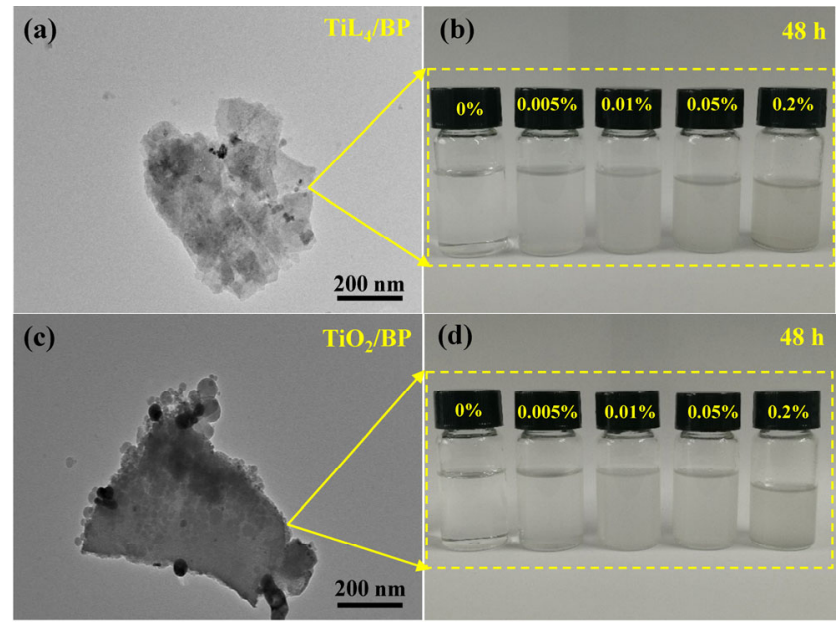

Fig. 3 TEM images of the nanocomposites and digital photos of PAO6 containing different mass concentrations of the nanocomposites after standing for $48 \mathrm{~h}$ : $(\mathrm{a}, \mathrm{b}) \mathrm{TiL}_{4} / \mathrm{BP}$, and $(\mathrm{c}, \mathrm{d})$ $\mathrm{TiO}_{2} / \mathrm{BP}$. containing or without BP nanocomposites under different loads. As can be observed from Fig. 4, with the introduction of $\mathrm{TiO}_{2} / \mathrm{BP}$ nanocomposites under different loads, all the average COF initially decreased before increasing. This trend is a result of the low concentrations of additives, forming a tribolayer to separate the friction pairs. Higher concentrations of additives may lead to aggregation [15], resulting in reduced lubricity. Compared with the BP nanosheets in previous work [15], the nanocomposites improved the tribological performance at high contact stress for steel/steel contact. $\mathrm{TiL}_{4} / \mathrm{BP}$ nanocomposites reduce friction up to a moderate load of $20 \mathrm{~N}$. This suggests either $\mathrm{TiL}_{4} / \mathrm{BP}$ nanocomposites do not form tribofilms under low pressure, or they cannot enter the sliding interface under high load [23].

Figure 5 shows the average wear scar diameter (WSD) of the upper ball under different lubrication conditions. As shown in Fig. 5, the WSD increased with the increasing load, which may have resulted from the destructive probability of asperities of the rubbing surfaces under higher pressure [24]. Compared with the pure PAO6, the PAO6 with nanocomposites had a smaller WSD under a moderate load of $20 \mathrm{~N}$, especially at the mass concentration of $0.01 \%$. Additionally, the lubricating effects of $\mathrm{TiO}_{2} / \mathrm{BP}$ nanocomposites increased compared to the $\mathrm{TiL}_{4} / \mathrm{BP}$ nanocomposites, which also agreed well with the results shown in Fig. 4. The following surface analysis will disclose the reason.

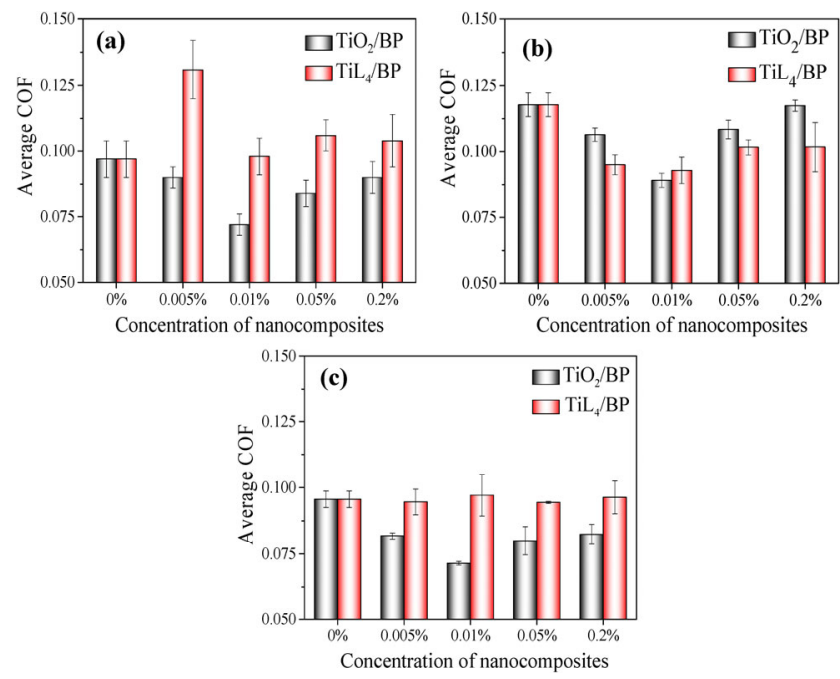

Fig. 4 Average COF of steel/steel contact lubricated by PAO6 containing or without BP nanocomposites under different loads: (a) $8 \mathrm{~N}$, (b) $20 \mathrm{~N}$, and (c) $30 \mathrm{~N}$. 

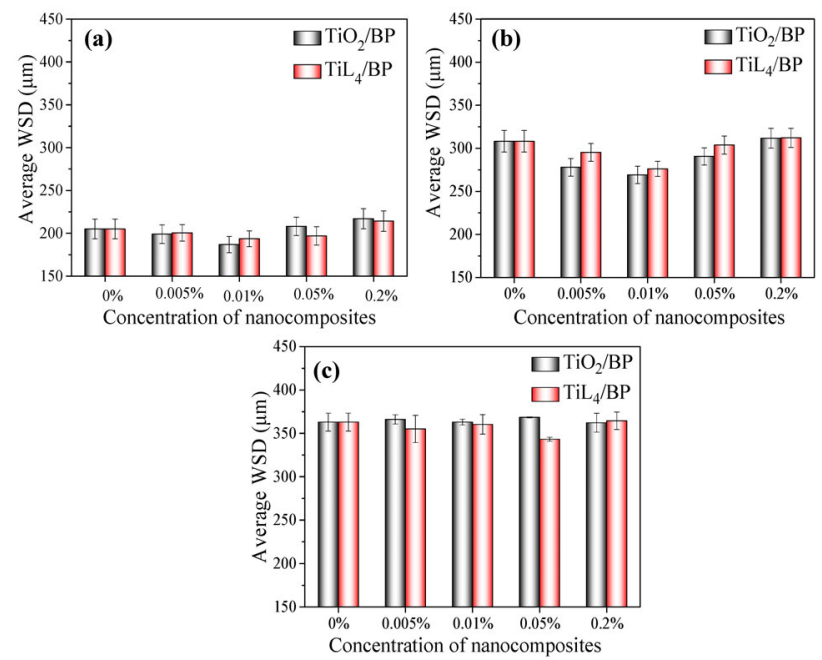

Fig. 5 Average WSD of the upper ball under different loads: (a) $8 \mathrm{~N}$, (b) $20 \mathrm{~N}$, and (c) $30 \mathrm{~N}$.

\subsection{Analysis of the worn surfaces}

Figure 6 depicts the wear profiles of the lower disk under PAO6 containing or without $\mathrm{TiL}_{4} / \mathrm{BP}$ nanocomposites. As can be seen from Fig. 6, with the increase of the loads, the wear depth and width of the lower disk increased, suggesting more severe wear. The concentration of $\mathrm{TiL}_{4} / \mathrm{BP}$ nanocomposites plays a critical role at the load of $20 \mathrm{~N}$, but the additive concentration had little effect on the WSD of the lower disk at lower or higher pressures, which is consistent with the wear result of the upper sample shown in Fig. 5.

Figure 7 shows the wear surface outlines of the lower disk lubricated by PAO6 containing or without
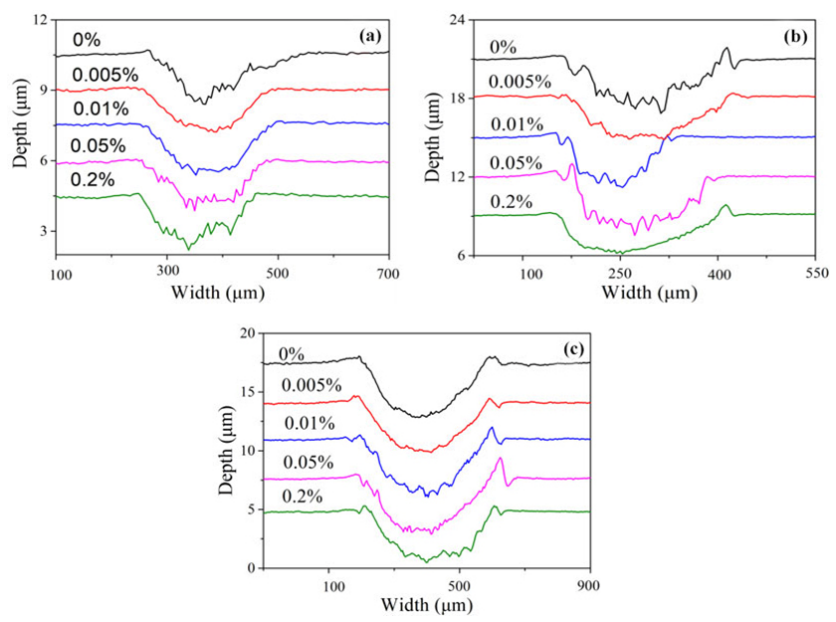

Fig. 6 Wear profiles of the lower disk lubricated by PAO6 containing or without $\mathrm{TiL}_{4} / \mathrm{BP}$ nanocomposites under different loads: (a) $8 \mathrm{~N}$, (b) $20 \mathrm{~N}$, and (c) $30 \mathrm{~N}$.
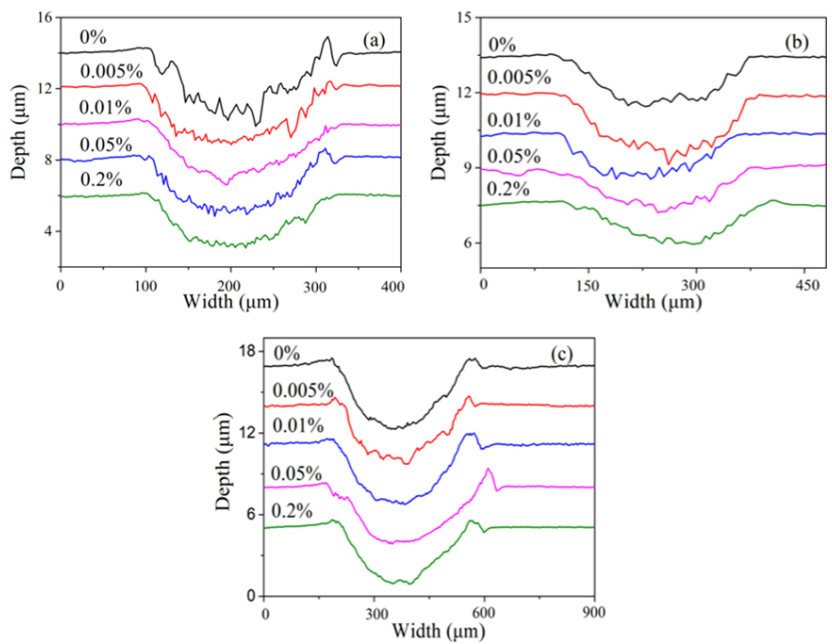

Fig. 7 Wear profiles of the lower disk lubricated by PAO6 containing or without $\mathrm{TiO}_{2} / \mathrm{BP}$ nanocomposites under different loads: (a) $8 \mathrm{~N}$, (b) $20 \mathrm{~N}$, and (c) $30 \mathrm{~N}$.

$\mathrm{TiO}_{2} / \mathrm{BP}$ nanocomposites. With the addition of $\mathrm{TiO}_{2} / \mathrm{BP}$ nanocomposites, the wear depth and width of the lower disk decreases. Furthermore, with the increase of nanocomposite contents in the lubricants, the size of the furrows on the specimen initially reduce before increasing. Under the same condition, the wear depth and width were smaller than those lubricated by PAO6 with $\mathrm{TiL}_{4} / \mathrm{BP}$ nanocomposites. This topology shows that the wear of the lower disk kept pace with those of the upper ball, which may be due to the friction pairs being of the same material.

Considering the dispersive effects and potential application, surface analysis of the specimens concentrated on those lubricated by PAO6 having mass concentrations less than $0.1 \%$. Figure 8 shows the SEM micrographs of the rubbed surfaces of the lower disk lubricated with PAO6 with different mass concentrations of $\mathrm{TiL}_{4} / \mathrm{BP}$ nanocomposites. Although using the same material in friction pairs could cause higher adhesive wear under high contact stress [25], no evident adhesive wear was observed in the SEM images owing to full lubrication. As can be seen, conspicuous dense furrows occurred at the worn surfaces at concentrations of $0.005 \%$ and $0.05 \%$, respectively. However, a relatively polished surface was noticed at the nanocomposite content of $0.01 \%$, suggesting that appropriate content of $\mathrm{BP}$ nanocomposites reduce friction and the wear of the sliding pairs. Some aggregation regions on the worn surface are evident at high concentrations, confirming former inferences. 


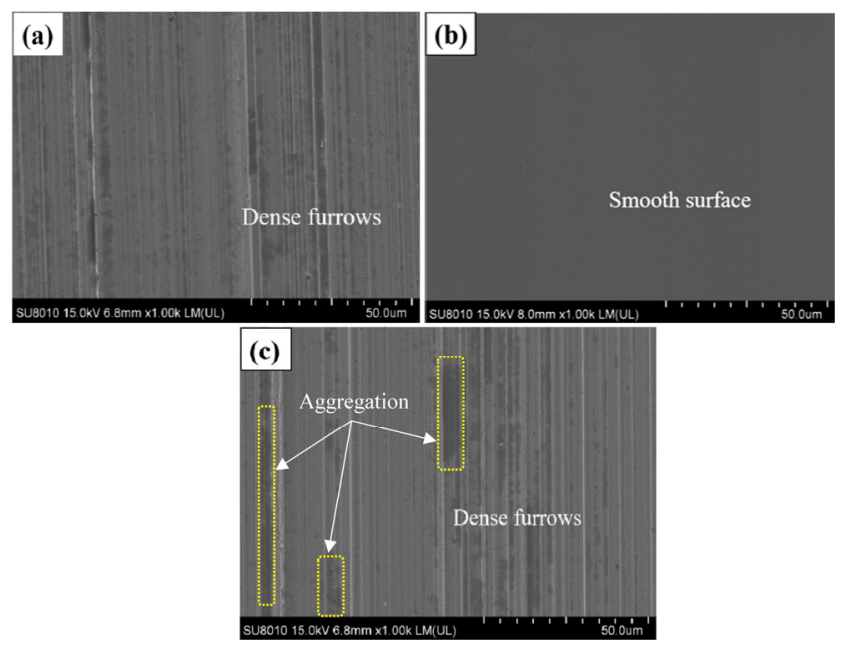

Fig. 8 SEM micrographs of the rubbed surfaces of the lower disk lubricated by PAO6 containing different mass concentrations of $\mathrm{TiL}_{4} / \mathrm{BP}$ nanocomposites under the load of $20 \mathrm{~N}$ : (a) $0.005 \%$, (b) $0.01 \%$, and (c) $0.05 \%$.

Figure 9 shows the SEM micrographs of the rubbed surfaces of the lower disk lubricated by PAO6 containing different concentrations of $\mathrm{TiO}_{2} / \mathrm{BP}$ nanocomposites. There are many pits, deep furrows, and cracks over the rubbed surfaces being lubricated by pure PAO6, as shown in Fig. 9(a), suggesting severe wear. With the addition of $\mathrm{TiO}_{2} / \mathrm{BP}$ nanocomposites, the worn surfaces became smoother and the furrows lighter, as shown in Figs. 9(b)-9(d), improved by the lubricity of the nanocomposites. The surfaces became smoothest at the concentration of $0.01 \%$. Higher levels

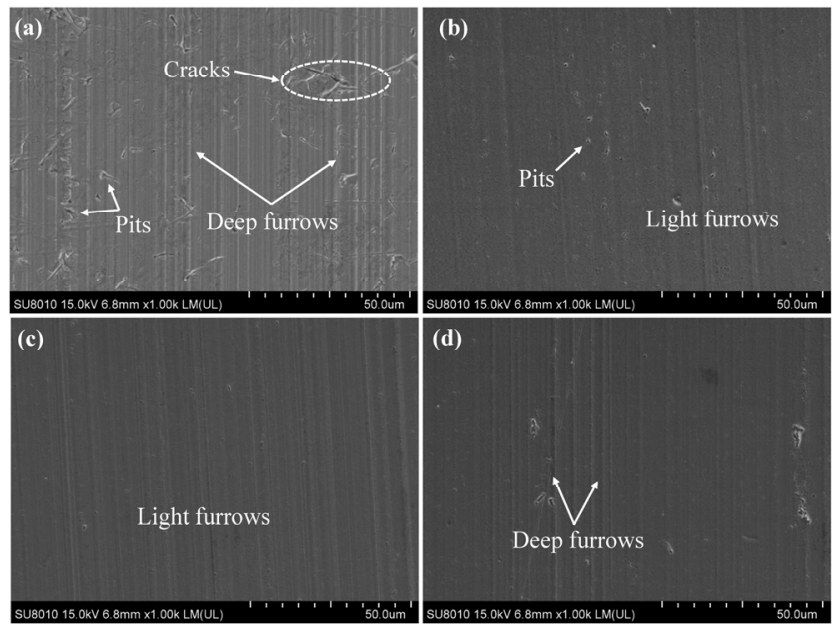

Fig. 9 SEM micrographs of the rubbed surfaces of the lower disk lubricated by PAO6 containing different mass concentrations of $\mathrm{TiO}_{2} / \mathrm{BP}$ nanocomposites under the load of $20 \mathrm{~N}$ : (a) $0 \%$, (b) $0.005 \%$, (c) $0.01 \%$, and (d) $0.05 \%$. of additive aggravated the surface roughness owing to the aggregation of the nanocomposites [26]. The worn surfaces displayed no aggregated regions, suggesting removal of the nanocomposites from the interface as a result of the 'ball-bearing' characteristics of nano- $\mathrm{TiO}_{2}$ [27].

The Raman spectra of the rubbed surfaces after sliding (Fig. 10) show two specific Raman peaks at approximately 250 and $670 \mathrm{~cm}^{-1}$, respectively, with the introduction of nanocomposites. These two peaks belong to $\mathrm{FeOOH}$ and $\mathrm{Fe}_{3} \mathrm{O}_{4}$, respectively [28]. These suggest that the addition of nanocomposites helped to generate an oxidation film on the sliding surfaces, reducing friction and wear [29]. However, these two peaks are small at the concentration of $0.01 \%$, where the average COF and WSD were lower. This can be demonstrated by the fact that the tribofilm dominates tribological behavior of the steel/steel contact and not the oxidation film.

To accurately discern the chemical components of the tribofilm, XPS spectra of the rubbed surfaces of the lower disk were detected. Figure 11 shows the XPS spectra of the rubbed surfaces lubricated with PAO6 containing or without $\mathrm{TiL}_{4} / \mathrm{BP}$ nanocomposites. As can be seen from Fig. 11(a), full-spectrum scanning detected three specific peaks of $\mathrm{C} 1 \mathrm{~s}, \mathrm{O} 1 \mathrm{~s}$, and Fe 2p. However, the expected P $2 p$ and Ti $2 p$ characteristic peaks were not found, which may be due to low concentrations on the worn surfaces. Moreover, the nanocomposite content of $0.01 \%$ had the highest intensity of $C$ 1s shown in Fig. 11(a), indicating the formation of the thickest adsorbed film from the base oil. This peak is ratified by the lowest Fe $2 p$ intensity, shown in Fig. 11(b), suggesting that the thickest adsorbed film covered the substrate steel surface [30]. These trends may be one reason for the best antifriction
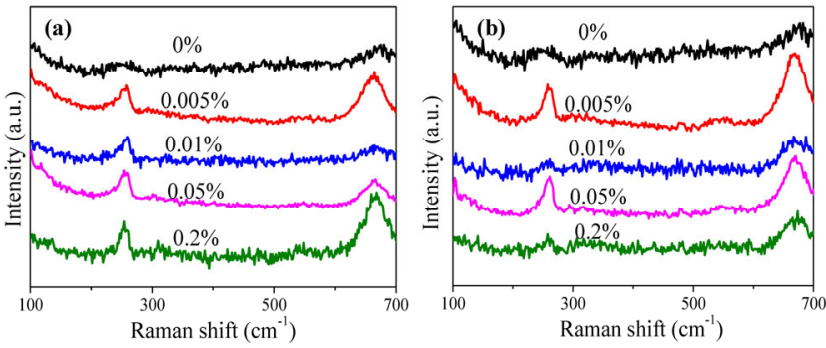

Fig. 10 Raman spectra of the rubbed surfaces lubricated by PAO6 containing different mass concentrations of nanocomposites under the load of $20 \mathrm{~N}$ : (a) $\mathrm{TiO}_{2} / \mathrm{BP}$ and (b) $\mathrm{TiL}_{4} / \mathrm{BP}$. 

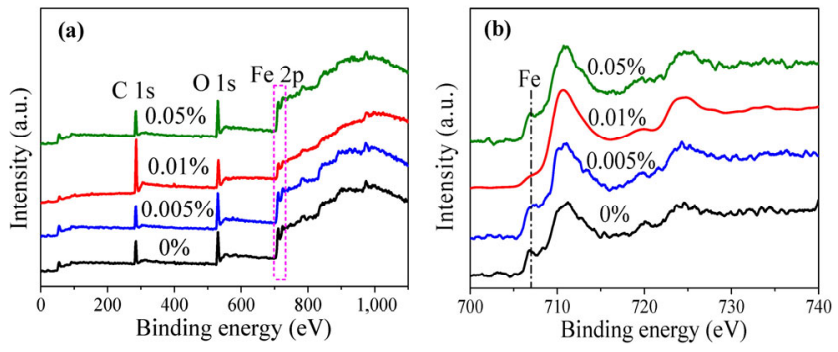

Fig. 11 XPS spectra of the rubbed surfaces lubricated by PAO6 containing or without $\mathrm{TiL}_{4} / \mathrm{BP}$ nanocomposites under the load of 20 N: (a) Survey and (b) Fe 2p.

and antiwear roles at this concentration for $\mathrm{TiL}_{4} / \mathrm{BP}$ nanocomposites as additives.

The $C$ 1s spectra of the rubbed surface lubricated with PAO6 containing different mass concentrations of $\mathrm{TiL}_{4} / \mathrm{BP}$ nanocomposites (Fig. 12) split into three components at approximately 285 (peak 1), 285.5 (peak 2), and $289 \mathrm{eV}$ (peak 3), corresponding to the $\mathrm{C}-\mathrm{C} / \mathrm{H}, \mathrm{C}-\mathrm{O}$, and $\mathrm{C}=\mathrm{O}$ chemical bonds, respectively [31]. The pure PAO6 lubricated surface had a higher content of $\mathrm{C}-\mathrm{C} / \mathrm{H}$, indicating that the adsorbed molecules mainly covered the sliding surface from the PAO6. With the introduction of $\mathrm{TiL}_{4} / \mathrm{BP}$ nanocomposites the area of peak 2 and 3 increased and peak 1 decreased, indicating that the content of the oxide film and tribofilm increased accordingly. The content of $\mathrm{C}-\mathrm{O}$ was highest when the concentration was $0.01 \%$, suggesting the thickest tribofilm over the rubbing
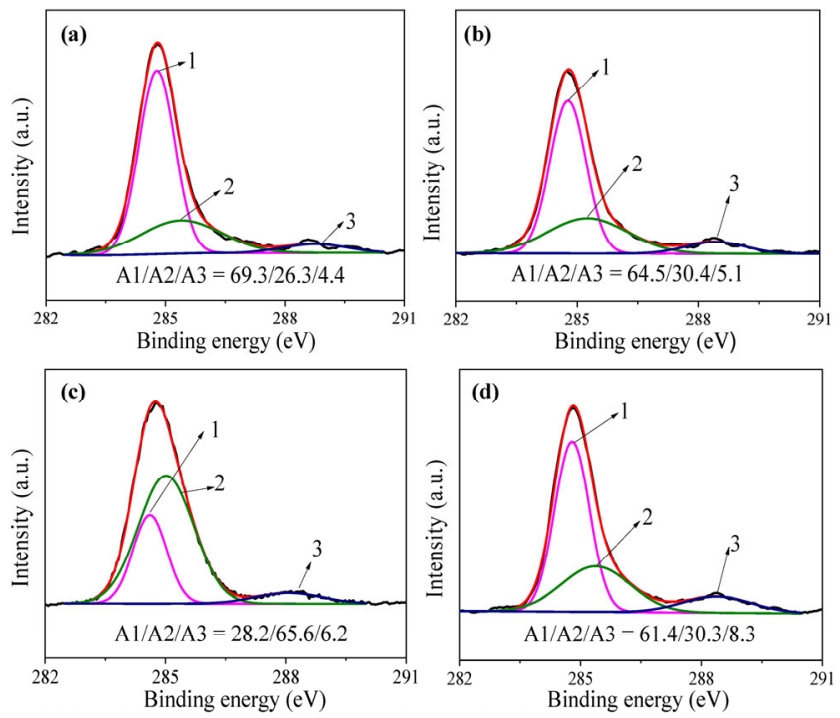

Fig. 12 XPS spectra of $C 1$ s of the rubbed surfaces lubricate by PAO6 containing different mass concentrations of $\mathrm{TiL}_{4} / \mathrm{BP}$ nanocomposites under the load of $20 \mathrm{~N}$ : (a) $0 \%$, (b) $0.005 \%$, (c) $0.01 \%$, and (d) $0.05 \%$. surface, which was helpful for their antifriction and antiwear roles [31].

Figure 13 shows the O 1s XPS spectra of the rubbed surface lubricated by PAO6 containing different mass concentrations of $\mathrm{TiL}_{4} / \mathrm{BP}$ nanocomposites. For the steel surfaces lubricated with pure PAO6, the peaks (1-3) at approximately 529.7, 530.3, and $\sim 531.4 \mathrm{eV}$ belonged to $\mathrm{Fe}_{3} \mathrm{O}_{4}$, or $\mathrm{FeOOH}, \mathrm{C}-\mathrm{O}$, and $\mathrm{C}=\mathrm{O}$ [31-33], respectively. The same oxide components detected by the Raman spectra shown in Fig. 10, confirming the formation of an oxide layer on the sliding surfaces. With the addition of $\mathrm{TiL}_{4} / \mathrm{BP}$ nanocomposites, two different peaks (4 and 5) located at approximately 532.9 and $535 \mathrm{eV}$ occurred. These belonged to $\mathrm{FePO}_{4}$ and $\mathrm{FeSO}_{4}$, respectively [34,35]. That is, the nanocomposites participated in a tribo-reaction and formed the film, which contributed to the lubrication. Furthermore, there was a higher content of $\mathrm{FePO}_{4}$ measured at the nanocomposite content of $0.01 \%$, which accounted for the better antifriction and antiwear performance [32].

Figure 14 depicts the XPS spectra of the rubbed surfaces lubricated by PAO6 containing or without $\mathrm{TiO}_{2} / \mathrm{BP}$ nanocomposites under the load of $20 \mathrm{~N}$. As can be seen from Fig. 14(a), full-spectrum scanning detected three specific peaks of $\mathrm{C} 1 \mathrm{~s}, \mathrm{O} 1 \mathrm{~s}$, and Fe 2p. Meanwhile, the expected P $2 p$ and Ti $2 p$ characteristic peaks were missing, which was due to the low content of the components on the rubbed surfaces.
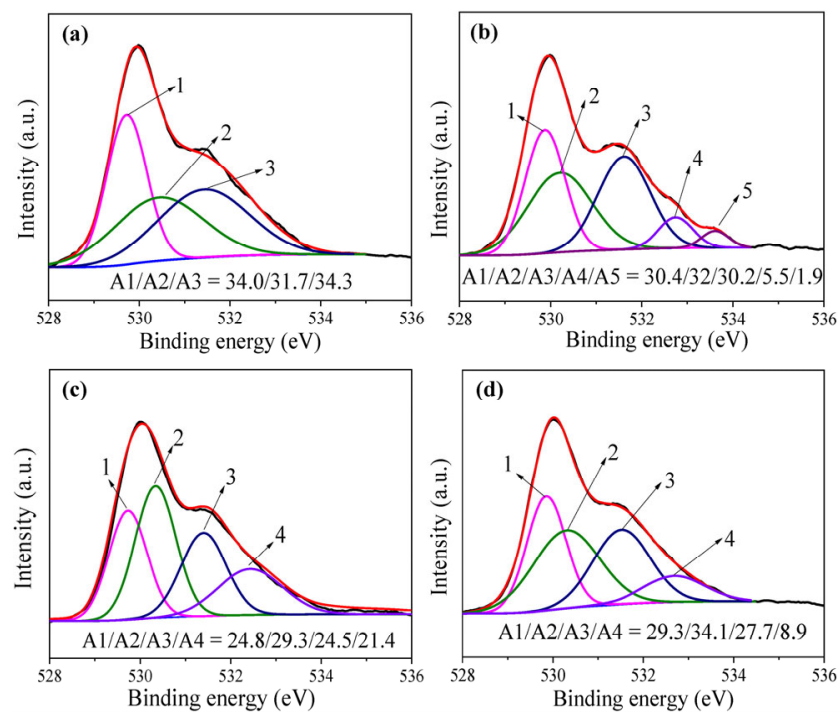

Fig. 13 XPS spectra of $O 1$ s of the rubbed surfaces lubricate by PAO6 containing different mass concentrations of $\mathrm{TiL}_{4} / \mathrm{BP}$ nanocomposites under the load of $20 \mathrm{~N}$ : (a) $0 \%$, (b) $0.005 \%$, (c) $0.01 \%$, and (d) $0.05 \%$. 

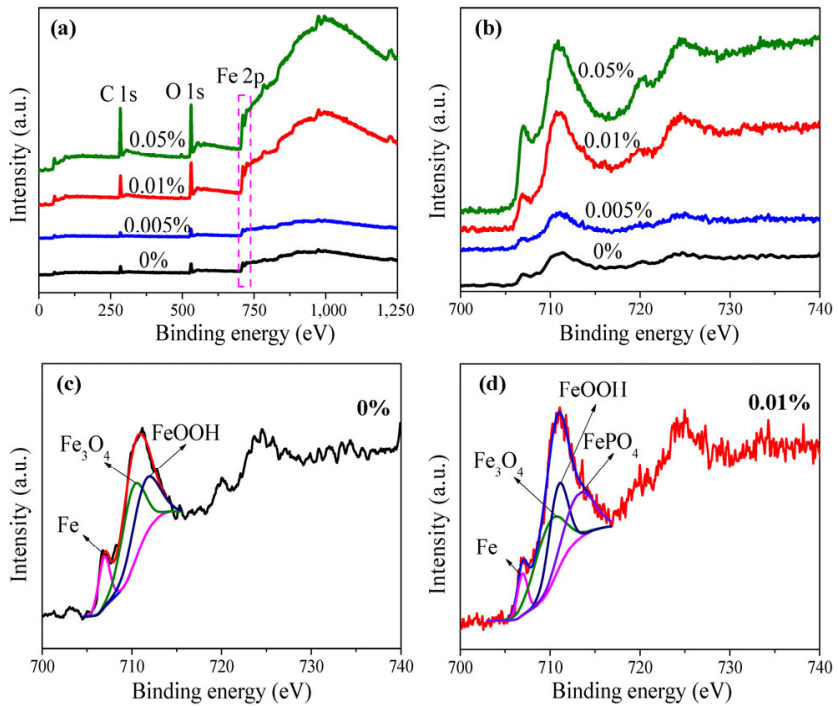

Fig. 14 XPS spectra of the rubbed surfaces lubricated by PAO6 containing or without different mass concentrations of $\mathrm{TiO}_{2} / \mathrm{BP}$ nanocomposites under the load of $20 \mathrm{~N}$ : (a) Survey, (b) Fe $2 \mathrm{p}$, (c) Fe $2 \mathrm{p}$ at $0 \%$, and (d) $\mathrm{Fe} 2 \mathrm{p}$ at $0.01 \%$.

Additionally, the 'ball-bearing' effects of nano- $\mathrm{TiO}_{2}$ [27] and the interlaminar slip between BP nanosheets [11] also contributed to the lubrication and decreased the concentration of $\mathrm{P} 2 \mathrm{p}$ and $\mathrm{Ti} 2 \mathrm{p}$ on the rubbed surfaces. Furthermore, with the concentration of the nanocomposites, reaching $0.01 \%$ and $0.05 \%$, both the carbon and oxygen content on the rubbed surface was much larger than other levels. This spectra shows that the introduction of $\mathrm{TiO}_{2} / \mathrm{BP}$ nanocomposites was good for the establishment of the adsorbing film, which has a different role than that of the $\mathrm{TiL}_{4} / \mathrm{BP}$ nanocomposites. At the same time, higher concentrations of $\mathrm{TiO}_{2} / \mathrm{BP}$ nanocomposites resulted in higher Fe $2 p$ content, which can be seen from Fig. 14(b). Additionally, for pure PAO6 lubrication (Fig. 14(c)), the main components in $\mathrm{Fe} 2 \mathrm{p}$ were $\mathrm{Fe}, \mathrm{Fe}_{3} \mathrm{O}_{4}$, and $\mathrm{FeOOH}$. However, high content of $\mathrm{FePO}_{4}$ was detected (Fig. 14(d)) in the tribofilm with the introduction of $\mathrm{TiO}_{2} / \mathrm{BP}$ nanocomposites at a concentration of $0.01 \%$, which favored better lubrication performance of the steel/steel contact at this condition [32].

Figure 15 shows the XPS spectrum of C 1s of the rubbed surface lubricated by PAO6 containing different mass concentrations of $\mathrm{TiO}_{2} / \mathrm{BP}$ nanocomposites under the load of $20 \mathrm{~N}$. As can be seen, the total C 1s spectra split into three peaks: peak 1 located at approximately $285 \mathrm{eV}$, assigned to the $\mathrm{C}-\mathrm{C}$ or $\mathrm{C}-\mathrm{H}$ vibrational peak; peak 2 located at $285-286 \mathrm{eV}$, attributed to the $\mathrm{C}-\mathrm{O}$
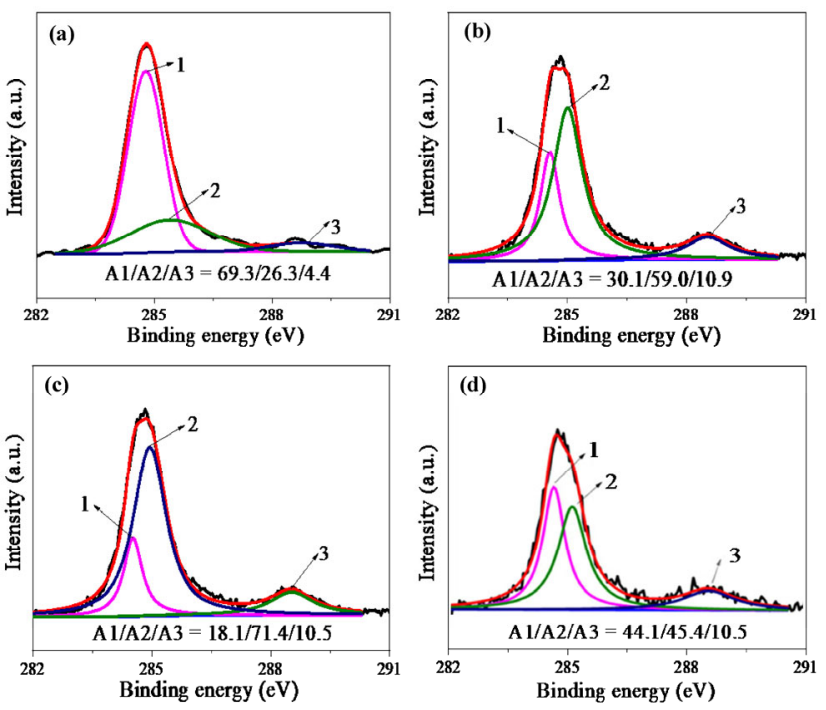

Fig. 15 XPS spectra of $\mathrm{C} 1 \mathrm{~s}$ of the rubbed surfaces lubricated by PAO6 containing different mass concentrations of $\mathrm{TiO}_{2} / \mathrm{BP}$ nanocomposites under the load of $20 \mathrm{~N}$ : (a) $0 \%$, (b) $0.005 \%$, (c) $0.01 \%$, and (d) $0.05 \%$.

vibration peak; peak 3 situated at approximately $289 \mathrm{eV}$, assigned to the $\mathrm{C}=\mathrm{O}$ vibration peak.

Pure PAO6 lubrication provided the lowest content of $\mathrm{C}-\mathrm{O}$ and $\mathrm{C}=\mathrm{O}$ peaks, indicating the thinnest oxide layer. With the introduction of $\mathrm{TiO}_{2} / \mathrm{BP}$ nanocomposites, a higher concentration of the oxide layer on the worn steel surface was obtained, especially at a levels of $0.01 \%$. These results confirm that the $\mathrm{TiO}_{2} / \mathrm{BP}$ nanocomposites are good for the formation of the oxide layer.

Figure 16 shows the XPS spectra of $\mathrm{O} 1 \mathrm{~s}$ of the rubbed surface lubricated by PAO6 containing different mass concentrations of $\mathrm{TiO}_{2} / \mathrm{BP}$ nanocomposites under the load of $20 \mathrm{~N}$. For rubbing surfaces lubricated by pure PAO6, shown in Fig. 16(a), peaks (1-3) positioned at 529.7, 530.3, and $531.4 \mathrm{eV}$ belonged to $\mathrm{Fe}-\mathrm{O}$ in $\mathrm{Fe}_{3} \mathrm{O}_{4}$ or $\mathrm{FeOOH}, \mathrm{C}-\mathrm{O}$, and $\mathrm{C}=\mathrm{O}$, respectively, which agreed well with the Raman spectra results. These spectra again support the view that the oxide layer forms by adsorption and frictional oxidation. When the $\mathrm{TiO}_{2} / \mathrm{BP}$ nanocomposites disperse in $\mathrm{PAO}$, a new peak (4) forms at $532.4 \mathrm{eV}$, attributed to $\mathrm{FePO}_{4}$. The relative peak area of $\mathrm{FePO}_{4}$ was highest at the $\mathrm{TiO}_{2} / \mathrm{BP}$ nanocomposites concentration of $0.01 \%$ indicating that the nanocomposites are adsorbed on the frictional surface and create a tribofilm, including $\mathrm{FePO}_{4}$, and playing a critical antifriction and antiwear role. 

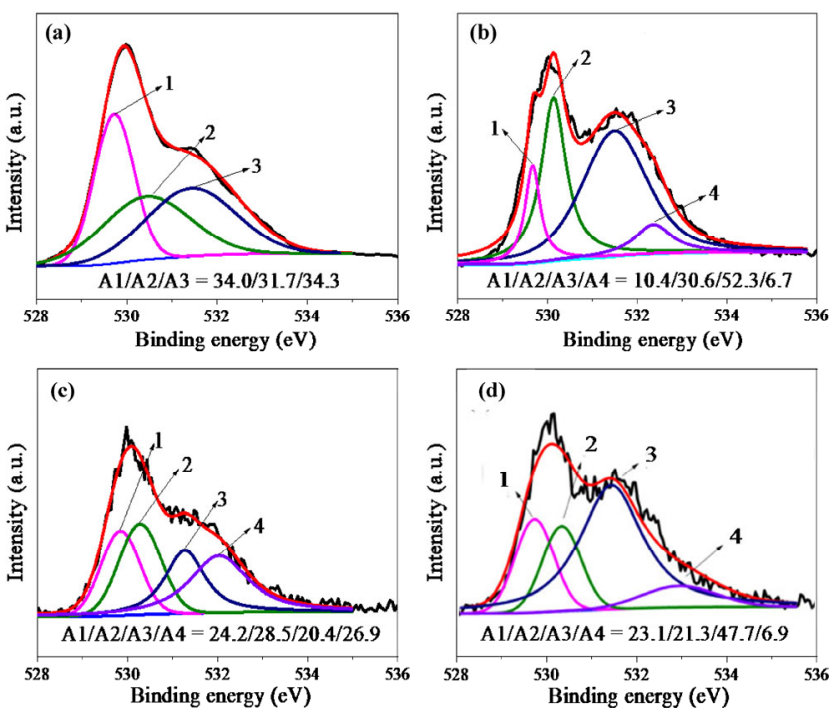

Fig. 16 XPS spectra of $\mathrm{O} 1 \mathrm{~s}$ of the rubbed surfaces lubricated by PAO6 containing different mass concentrations of $\mathrm{TiO}_{2} / \mathrm{BP}$ nanocomposites under the load of $20 \mathrm{~N}$ : (a) $0 \%$, (b) $0.005 \%$, (c) $0.01 \%$, and (d) $0.05 \%$.

The boundary lubrication process of the steel/steel friction pairs lubricated by the PAO6 containing BP nanocomposites additives is active, the mechanisms work as follows. The PAO6 and nanocomposites are adsorbed on the sliding interfaces and form an adsorption film, an oxide film, and a tribofilm during the frictional process. For the PAO6 lubricating process, the adsorption film containing organic molecules from PAO6 and the oxide film containing $\mathrm{Fe}_{3} \mathrm{O}_{4}$ or $\mathrm{FeOOH}$ play a friction-reducing and antiwear role, and the adsorption film mainly dominated the friction and wear behavior. During the PAO6 containing BP nanocomposites lubricating process, the tribofilm dominated the sliding process. However, excessive concentrations of nanocomposites do not make for optimum lubrication performance owing to aggregation. Under the same conditions, $\mathrm{TiO}_{2} / \mathrm{BP}$ nanocomposites have better lubricating effects than $\mathrm{TiL}_{4} / \mathrm{BP}$ nanocomposites, besides the formation of tribofilm, partially due to the synergistic effect of the bearing capacity, the rolling effects of nano- $\mathrm{TiO}_{2}$, and the interlaminar slip between BP nanosheets.

\section{Conclusions}

In summary, to reduce the friction and wear effects of steel/steel tribo-pairs, two types of BP nanocomposites were synthesized and dispersed in PAO6 as additives. A ball-on-disk tribometer configured to produce boundary lubrication conditions measured the tribological behavior of steel/steel contacts. Several advanced surface analysis techniques characterized the sliding surfaces. The primary conclusions obtained from this investigation are as follows:

1) Titanium dioxide with the size of $10-30 \mathrm{~nm}$ can be evenly deposited over the surface of BP nanosheets by the sol-gel method; and titanium sulfonate ligand can be successfully adsorbed outside the BP nanosheets to form $\mathrm{TiL}_{4} / \mathrm{BP}$ nanocomposites.

2) For steel/steel contact lubrication, the optimal mass concentration of these two BP nanocomposites in PAO6 is $0.01 \%$. Excessive high levels lead to aggregation of the additives and deterioration of the lubrication effects.

3) The addition of $\mathrm{TiL}_{4} / \mathrm{BP}$ nanocomposites improved the tribological performance of the steel/steel contact when compared with the BP nanosheets, especially under a load of $20 \mathrm{~N}$. Lower loads inhibited the formation of a tribofilm. In contrast, higher pressures decreased the interval between friction pairs and adversely limited the entrance of nanocomposites.

4) With the introduction of $\mathrm{TiO}_{2} / \mathrm{BP}$ nanocomposites, the worn steel surface changed from severe to slight wear. The tribological mechanism on the sliding surface was a combination of the bearing capacity and rolling effects of nano titanium dioxide, the formation of a tribofilm including $\mathrm{FePO}_{4}$, and the interlaminar slip of BP nanosheets.

\section{Acknowledgements}

The authors appreciate the financial support of the National Natural Science Foundation of China (Grant No. 51875155) and the Fundamental Research Funds for the Central Universities (Grant No. PA2019GDQT0017). In addition, professor Kunhong $\mathrm{HU}$ and associate professor Enzhu HU are gratefully acknowledged for providing half of the testing instruments and fruitful suggestions.

Open Access This article is licensed under a Creative Commons Attribution 4.0 International License, which permits use, sharing, adaptation, distribution and 
reproduction in any medium or format, as long as you give appropriate credit to the original author(s) and the source, provide a link to the Creative Commons licence, and indicate if changes were made.

The images or other third party material in this article are included in the article's Creative Commons licence, unless indicated otherwise in a credit line to the material. If material is not included in the article's Creative Commons licence and your intended use is not permitted by statutory regulation or exceeds the permitted use, you will need to obtain permission directly from the copyright holder.

To view a copy of this licence, visit http://creativecommons.org/licenses/by/4.0/.

\section{References}

[1] Huang G W, Yu Q L, Ma Z F, Cai M R, Zhou F, Liu W M. Oil-soluble ionic liquids as antiwear and extreme pressure additives in poly- $\alpha$-olefin for steel/steel contacts. Friction 7(1): 18-31 (2019)

[2] Hou K M, Wang J Q, Yang Z G, Ma L M, Wang Z F, Yang S R. One-pot synthesis of reduced graphene oxide/molybdenum disulfide heterostructures with intrinsic incommensurateness for enhanced lubricating properties. Carbon 115: 83-94 (2017)

[3] Sayuti M, Sarhan A A D, Salem F. Novel uses of $\mathrm{SiO}_{2}$ nano-lubrication system in hard turning process of hardened steel AISI4140 for less tool wear, surface roughness and oil consumption. J Clean Prod 67: 265-276 (2014)

[4] Xu Y F, Geng J, Peng Y B, Liu Z C, Yu J Y, Hu X G. Lubricating mechanism of $\mathrm{Fe}_{3} \mathrm{O}_{4} @ \mathrm{MoS}_{2}$ core-shell nanocomposites as oil additives for steel/steel contact. Tribol Int 121: 241-251 (2018)

[5] Zeng Y M, He F, Wang Q, Yan X H, Xie G X. Friction and wear behaviors of molybdenum disulfide nanosheets under normal electric field. Appl Surf Sci 455: 527-532 (2018)

[6] Mura A, Adamo F, Wang H Z, Leong W S, Ji X, Kong J. Investigation about tribological behavior of ABS and PC-ABS polymers coated with graphene. Tribol Int 134: 335-340 (2019)

[7] Morina A, Neville A, Priest M, Green J H. ZDDP and MoDTC interactions in boundary lubrication-The effect of temperature and ZDDP/MoDTC ratio. Tribol Int 39: 1545-1557 (2006)

[8] Morina A, Neville A, Green J H, Priest M. Additive/ additive interactions in boundary lubrication-A study of film formation and tenacity. Tribol Interface Eng Ser 48:
757-767 (2005)

[9] Dawczyk J, Morgan N, Russo J, Spikes H. Film thickness and friction of ZDDP tribofilms. Tribol Lett 67(2): 34 (2019)

[10] Komvopoulos K, Do V, Yamaguchi E S, Ryason P R. Nanomechanical and nanotribological properties of an antiwear tribofilm produced from phosphorus-containing additives on boundary-lubricated steel surfaces. J Tribol 126(4): 775-780 (2004)

[11] Wang W, Xie G X, Luo J B. Black phosphorus as a new lubricant. Friction 6(1): 116-142 (2018)

[12] Lv Y, Wang W, Xie G X, Luo J B. Self-lubricating PTFEbased composites with black phosphorus nanosheets. Tribol Lett 66(2): 61 (2018)

[13] Chen W S, Ouyang J, Yi X Y, Xu Y, Niu C C, Zhang W Y, Wang L Q, Sheng J P, Deng L, Liu Y N, et al. Black phosphorus nanosheets as a neuroprotective nanomedicine for neurodegenerative disorder therapy. Adv Mater 30(3): 1703458 (2018)

[14] Yuan S F, Shen C F, Deng B C, Chen X L, Guo Q S, Ma Y Q, Abbas A, Liu B L, Haiges R, Ott C, et al. Air-stable room-temperature mid-infrared photodetectors based on hBN/black arsenic phosphorus/hBN heterostructures. Nano Lett 18(5): 3172-3179 (2018)

[15] Xu Y F, Yu J Y, Dong Y H, You T, Hu X G. Boundary lubricating properties of black phosphorus nanosheets in polyalphaolefin oil. J Tribol 141(7): 072101 (2019)

[16] Bogunovic L, Zuenkeler S, Toensing K, Anselmetti D. An oil-based lubrication system based on nanoparticular $\mathrm{TiO}_{2}$ with superior friction and wear properties. Tribol Lett 59(2): 29 (2015)

[17] Meng Y N, Sun J L, Wu P, Dong C, Yan X D. The Role of nano- $\mathrm{TiO}_{2}$ lubricating fluid on the hot rolled surface and metallographic structure of SS41 Steel. Nanomaterials 8(2): 111 (2018)

[18] Poornima V P, Mariam A S. $\mathrm{TiO}_{2}$ nanotubes and mesoporous silica as containers in self-healing epoxy coatings. Sci Rep 6(1): 38812 (2016)

[19] Lee H U, Lee S C, Won J, Son B C, Choi S, Kim Y, Park S Y, Kim H S, Lee Y C, Lee J. Stable semiconductor black phosphorus (BP)@titanium dioxide $\left(\mathrm{TiO}_{2}\right)$ hybrid photocatalysts. Sci Rep 5: 8691 (2015)

[20] Zhao Y T, Wang H Y, Huang H, Xiao Q L, Xu Y H, Guo Z N, Xie H H, Shao J D, Sun Z B, Han W J, et al. Surface coordination of black phosphorus for robust air and water stability. Angew Chem 128(16): 5087-5091 (2016)

[21] Li L K, Yu Y J, Ye G J, Ge Q Q, Ou X D, Wu H, Feng D L, Chen X H, Zhang Y B. Black phosphorus field-effect transistors. Nat Nanotechnol 9(5): 372-377 (2014)

[22] Zhang J, Li M J, Feng Z C, Chen J, Li C. UV Raman 
spectroscopic study on $\mathrm{TiO}_{2}$. I. Phase transformation at the surface and in the bulk. $J$ Phy Chem B 110(2): 927-935 (2006)

[23] Podgornik B, Jacobson S, Hogmark S. Influence of EP additive concentration on the tribological behaviour of DLCcoated steel surfaces. Surf Coat Technol 191(2-3): 357-366 (2005)

[24] Kumar N, Gautam G, Gautam R K, Mohan A, Mohan S. Wear, friction and profilometer studies of insitu AA5052/ $\mathrm{ZrB}_{2}$ composites. Tribol Int 97: 313-326 (2016)

[25] Okonkwo P C, Kelly G, Rolfe B F, Pereira M P. The effect of sliding speed on the wear of steel-tool steel pairs. Tribol Int 97: 218-227 (2016)

[26] Wu D Y, Xu Y F, Yao L L, You T, Hu X G. Tribological behaviour of graphene oxide sheets as lubricating additives in bio-oil. Ind Lub Tribol 70(8): 1396-1401 (2018)

[27] Sun J L, Zhu Z X, Xu P F. Study on the lubricating performance of nano- $\mathrm{TiO}_{2}$ in water-based cold rolling fluid. Mater Sci Forum 817: 219-224 (2015)

[28] Tiwari S, Phase D M, Choudhary R J. Probing antiphase boundaries in $\mathrm{Fe}_{3} \mathrm{O}_{4}$ thin films using micro-Raman spectroscopy. Appl Phys Lett 93(23): 234108 (2008)

[29] Chen L, Jin X Y, Qu Y, Wei K J, Zhang Y F, Liao B, Xue $\mathrm{W}$ B. High temperature tribological behavior of microarc

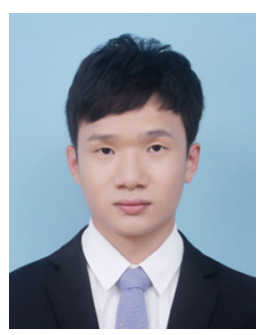

Zhiheng LUO. He received his bachelor degree in 2018 from Jiangxi Agricultural University, China. Then he was a graduate student in

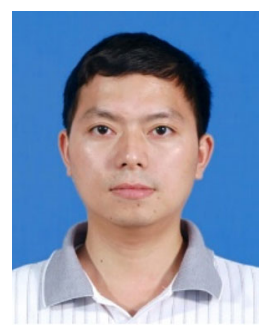

Yufu XU. He received his bachelor, master, and Ph.D. degrees from Hefei University of Technology, China, in 2003, 2006, and 2012, respectively. He joined the Institute of Tribology at Hefei University of Technology from 2006. He spent one year (2015-2016) in the Tribology laboratory, University oxidation film on Ti-39Nb-6Zr alloy. Surf Coat Technol 347: 29-37 (2018)

[30] Xu Y F, Yu J Y, Geng J, Abuflaha R, Olson D, Hu X G, Tysoe W T. Characterization of the tribological behavior of the textured steel surfaces fabricated by photolithographic etching. Tribol Lett 66(2): 55 (2018)

[31] Zhao H Y, Morina A, Neville A, Vickerman R. Tribochemistry on clutch friction material lubricated by automatic transmission fluids and the link to frictional performance. $J$ Tribol 135(4): 041801 (2013)

[32] Yu Q L, Zhang C Y, Dong R, Shi Y J, Wang Y R, Bai Y Y, Zhang J Y, Cai M R, Zhou F. Novel N,P-containing oilsoluble ionic liquids with excellent tribological and anticorrosion performance. Tribol Int 132: 118-129 (2019)

[33] Nan F, Zhou K H, Liu S, Pu J B, Fang Y H, Ding W X. Tribological properties of attapulgite $/ \mathrm{La}_{2} \mathrm{O}_{3}$ nanocomposite as lubricant additive for a steel/steel contact. RSC $A d v \mathbf{8}(30)$ : 16947-16956 (2018)

[34] Yang Y, Zhang C H, Wang Y, Dai Y J, Luo J B. Friction and wear performance of titanium alloy against tungsten carbide lubricated with phosphate ester. Tribol Int 95: 27-34 (2016)

[35] Zhang S W, Liu H C, He R Y. Mechanisms of wear of steel by natural rubber in water medium. Wear 256(3-4): 226-232 (2004)

mechanical engineering of the Institute of Tribology at Hefei University of Technology. His research interests include the tribology of nano-lubricating additives and wear of coatings.

of Wisconsin-Milwaukee as a visiting scholar at Wilfred T. Tysoe's group sponsored by China Scholarship Council. He has published more than 40 peer-reviewed papers that received more than 1,000 citations and has the h-index 20. His current position is a professor. His research interests cover the tribology of nanoparticles and bioenergy, smart lubrication, and active control of friction and wear. 